\title{
Synthesis of intelligent fuzzy control in the space of bounded piecewise-continuous functions based on the combined maximum principle
}

\author{
Andrey A. Kostoglotov ${ }^{1}$, Sergey $V$. Lazarnko ${ }^{2, *}$, and Igor A. Nikitin ${ }^{3}$ \\ ${ }^{1}$ Rostov State Transport University, Department of Communication on Railway Transport, \\ Rostov-on-Don, Russian Federation \\ ${ }^{2}$ Don State Technical University, Department of Radio Engineering and Electronics, Rostov-on-Don, \\ Russian Federation \\ ${ }^{3}$ Moscow State University of Technology and Management named after K.G. Razumovsky (the First \\ Cossacs University), Department of Technology of Gain Processing, Bread, Macaroni and \\ Confectionery Productions, Moscow, Russian Federation
}

\begin{abstract}
It is shown that the solution of the problem of control synthesis using the Hamilton-Ostrogradskii principle leads to a variational inequality, from which the conditions for the maximum of the the generalized power function in the space of bounded piecewise continuous functions follow. It allows to find a feedback structure up to a synthesis function. Using the methods of the structure construction the nonlinear structures of the relay and continuous control laws are obtained. The proposed control method allows to avoid the mode with frequented switching. It consists of the following two stages. At the first stage, the control object is brought into the vicinity of the terminal state using the relay control law. In the second stage, the quasi-optimal continuous control is used. The uncertainty of the transition area size is resolved using fuzzy logic. The efficiency of the intelligent controls is demonstrated by the example of mathematical modeling of the system dynamics.
\end{abstract}

\section{Introduction}

The central result, extending the calculus of variations, is the optimality principle of L.S. Pontryagin, which can be used to calculate program control of both linear and nonlinear systems [1]. However, the construction of the optimal control for nonlinear systems is a very complicated problem even for the case of piecewise-constant controls that lead to the appearance of the chattering modes $[1,2]$. The reason for this situation is that on the optimal trajectory the Hamiltonian maximum is reached at more than one point. The frequented switching mode is an obstacle for the implementation of such controls in real technical systems. This gave rise to a large number of methods for synthesizing controls in the region of the sliding mode $[2,3]$.

\footnotetext{
*Corresponding author: lazarenkosv@icloud.com
} 
It is shown in this paper that the solution of the problem of the controls synthesis in the space of bounded piecewise continuous functions using the Hamilton-Ostrogradskii principle [4] leads to the maximum condition for the function of generalized power [5 - 8]. Its application makes it possible to construct feedback structures on the boundary of the domain of admissible controls and within the boundary. The proximity of the synthesized quasi-optimal intelligent fuzzy controls to the optimal solution is confirmed by the results of mathematical modeling using the example of solving the performance problem.

\section{Formulation of the problem}

Let us consider a holonomic controlled dynamical system which action integral has the form [4]

$$
\mathrm{S}=\int_{t_{0}}^{t_{1}}(T+A) d t
$$

where $T=\frac{1}{2} \sum_{s, k=1}^{n} a_{s k}(\mathbf{q}, t) \dot{q}_{s} \dot{q}_{k}-$ is kinetic energy; $a_{s k}(\mathbf{q}, t)$ - are the bounded inertia coefficients; $\quad \mathbf{q}=\left[q_{1}, \ldots q_{n}\right]^{\mathrm{T}} \in R^{n} \quad-$ is the vector of generalized coordinates; $A=\sum_{s=1}^{n} \int_{q_{s}\left(t_{0}\right)}^{q_{s}\left(t_{1}\right)} Q_{s} d q_{s}-$ is the work of generalized forces; $\mathbf{Q}=\left[Q_{1}(\mathbf{q}, \dot{\mathbf{q}}, t), \ldots, Q_{n}(\mathbf{q}, \dot{\mathbf{q}}, t)\right]^{\mathrm{T}}-$ is vector of generalized forces continuous in the set of variables, due to the presence of various kinds of external and internal effects [9]; $q_{s}\left(t_{0}\right), q_{s}\left(t_{1}\right)$ - are initial and final states; $n=\operatorname{dim} \mathbf{q}-$ is the number of degrees of freedom of the dynamical system; $\mathrm{T}-$ is sign of transposition; the dot denotes the time derivative; $\left[t_{0}, t_{1}\right] \subset R-$ is given time interval.

In accordance with the Hamilton - Ostrogradsky principle, when the system moves from the initial state $t=t_{0}, \mathbf{q}\left(t_{0}\right)=\left[q_{10}, \ldots q_{n 0}\right]^{\mathrm{T}}, \dot{\mathbf{q}}\left(t_{0}\right)=\left[\dot{q}_{10}, \ldots \dot{q}_{n 0}\right]^{\mathrm{T}}$ to the final state $t=t_{1}, \mathbf{q}\left(t_{1}\right)=\left[q_{11}, \ldots q_{n 1}\right]^{\mathrm{T}}, \dot{\mathbf{q}}\left(t_{1}\right)=\left[\dot{q}_{11}, \ldots \dot{q}_{n 1}\right]^{\mathrm{T}}$ under the action of generalized forces on the true trajectory, the stationarity principle for the integral of the action (1) is true [4]

$$
\delta^{\prime} \mathrm{S}=\int_{t_{0}}^{t_{1}}\left(\delta T+\delta^{\prime} A\right) d t=0
$$

where $\delta^{\prime} A=\sum_{s=1}^{n} Q_{s} \delta q_{s}-$ is the elementary work on virtual movements, and the sign $\delta^{\prime}$ denotes an infinitesimal value [4].

Equation (4) implies the Lagrange equations of the second kind

$$
\frac{d}{d t}\left(\frac{\partial T}{\partial \dot{q}_{s}}\right)-\frac{\partial T}{\partial q_{s}}=Q_{s}, \quad s=\overline{1, n,}
$$

where the control actions are identified as the generalized forces. Such a case "plays a central role in the consideration of the systems of the form (3) and their generalizations" [9].

Let us define on a bounded closed time interval $\left[t_{0}, t_{1}\right] \subset R$ a scalar continuous positivedefinite target function $\mathrm{F}(\mathbf{q})$ with its partial derivatives. The problem of control synthesis is 
posed as the inverse problem of dynamics: among admissible generalized forces $\mathbf{Q} \in \bar{G}$ it is required to find one for which the target functional

$$
\mathrm{I}(\mathbf{q})=\int_{t_{0}}^{t_{1}} \mathrm{~F}(\mathbf{q}) d t \rightarrow \min _{\mathbf{Q} \in \bar{G}}
$$

takes the smallest possible value; here $\bar{G}$ is the region of admissible generalized forces.

\section{Conditions for the maximum of the function of generalized power in the space of bounded piecewise continuous functions}

Let us consider the extended target functional [5-8]

$$
\mathrm{I}_{1}(\mathbf{q}, \mathbf{Q})=\mathrm{I}(\mathbf{q})+\lambda \mathrm{S}(\mathbf{Q}),
$$

where $\lambda-$ is the Lagrange multiplier.

Suppose that there exists the vector of generalized forces $\widetilde{\mathbf{Q}} \in \bar{G}$ and the corresponding trajectory $\widetilde{\mathbf{q}}$ that delivers a minimum of (4). Variation of the generalized force leads to the vector $\mathbf{Q}=\widetilde{\mathbf{Q}}+\delta \mathbf{Q}$ to which the motion along a roundabout path corresponds, and $\delta \mathbf{Q}$ is a variation such as $\mathbf{Q} \in \bar{G}$. Then the following inequality holds:

$$
\mathrm{I}_{1}(\mathbf{q}, \mathbf{Q})-\mathrm{I}_{1}(\widetilde{\mathbf{q}}, \widetilde{\mathbf{Q}}) \geq 0, \quad \forall \mathbf{Q} \neq \widetilde{\mathbf{Q}}
$$

We consider the case when a point $\widetilde{q}_{s}(t)$ on the initial trajectory is mapped to the point $q_{s}(t+\Delta t)=\widetilde{q}_{s}(t)+\Delta q_{s}(t)$ of the variated trajectory; here $\Delta t$ is an arbitrary non-negative infinitesimal time function, and $\Delta q_{s}$ is an asynchronous (full) coordinate variation. Let us consider the asynchronous variation of the extended target functional $[5-8]$

$$
\Delta \mathrm{I}_{1}=\mathrm{I}_{1}(\mathbf{q}, \mathbf{Q})-\mathrm{I}_{1}(\widetilde{\mathbf{q}}, \widetilde{\mathbf{Q}})=\left.[\lambda(T+A)+\mathrm{F}] \Delta t\right|_{t_{0}} ^{t_{1}}+\delta \mathrm{I}_{1},
$$

where $\delta \mathrm{I}_{1}$ - is the main part of the extended target functional increment. Since $\Delta t\left(t_{1}\right), \Delta t\left(t_{0}\right)$ are not equal to zero and independent, we find the stationary value of the extended target functional $\mathrm{I}_{1}$ containing the indefinite Lagrange multiplier. The factor at $\Delta t\left(t_{1}\right), \Delta t\left(t_{0}\right)$ should be assumed to be zero [4-8]. From this it follows the transversality condition

$$
[\lambda(T+A)+\mathrm{F}]_{t_{0}}^{t_{1}}=0 .
$$

The target functional increment

$$
\Delta \mathrm{I}_{1}=\int_{t_{0}}^{t_{1}} \sum_{s=1}^{n}\left[\lambda \delta Q_{s}+\delta V_{s}\right] \delta q_{s} d t+\int_{t_{0}}^{t_{1}} \sum_{s=1}^{n} \widetilde{V}_{s} \delta q_{s} d t
$$

The increment of a continuous positive-definite target functional on a trajectory that delivers an extremum to the target functional is nonnegative, therefore $\int_{t_{0}}^{t_{1}} \sum_{s=1}^{n} \widetilde{V}_{s} \delta q_{s} d t \geq 0$. Then under the condition 


$$
\int_{t_{0}}^{t_{1}} \sum_{s=1}^{n}\left[\lambda \delta Q_{s}+\delta V_{s}\right] \delta q_{s} d t \geq 0
$$

the inequality (9) holds true. It is reasonable to carry out the investigation (10) for a specific set of admissible generalized forces (controls) [5-8].

Let the variation of the generalized force $|\mathbf{Q}| \leq \mathbf{C}$ be constructed as an needle variation

$$
\delta \mathbf{Q}(t)= \begin{cases}\mathbf{C}, & t \in[\tau, \tau+\varepsilon], \\ \widetilde{\mathbf{Q}}(t), & t \bar{\epsilon}[\tau, \tau+\varepsilon],\end{cases}
$$

where $\mathbf{C}$ is a vector of nonnegative constants, $\tau \in\left(t_{0}, t_{1}\right)$ is a time that specifies a closed time interval $[\tau, \tau+\varepsilon], \varepsilon>0$ is a small value. when

Taking into account the properties of the variations the expression (10) is nonnegative,

$$
\sum_{s=1}^{n}\left[\lambda \widetilde{Q}_{s}+\widetilde{V}_{s}\right] \widetilde{\dot{q}}_{s} \geq \sum_{s=1}^{n}\left[\lambda Q_{s}+V_{s}\right] \dot{q}_{s} .
$$

Then to achieve an extremum it is necessary that the function of generalized power

$$
\Phi=\sum_{s=1}^{n}\left[\lambda Q_{s}+V_{s}\right] \dot{q}_{s}
$$

reaches its maximum

$$
\max _{|\mathbf{Q}| \mathbf{C} \mathbf{C}} \Phi=\max _{|\mathbf{Q}| \leq \mathbf{C}} \sum_{s=1}^{n}\left[\lambda Q_{s}+V_{s}\right] \dot{q}_{s} .
$$

Let $\widetilde{\mathbf{Q}}$ belongs to the continuous vector functions class, $\widetilde{\mathbf{Q}} \in C^{n}\left[t_{0}, t_{1}\right]$. The variation of the generalized force has the form

$$
\delta \mathbf{Q}(t)=\left\{\begin{array}{l}
\mathbf{v}(t), \quad t \in[\tau, \tau+\varepsilon], \\
\widetilde{\mathbf{Q}}(t), t \bar{\epsilon}[\tau, \tau+\varepsilon],
\end{array}\right.
$$

where $\tau \in\left(t_{0}, t_{1}\right)$ is an arbitrary point of continuity of the vector function $\delta \mathbf{Q} \in C^{n}\left[t_{0}, t_{1}\right]$, $\mathbf{v}(t)$ is a bounded vector function.

Then the integrand (10) is a real continuous function and, in accordance with [1], we have

$$
\sum_{s=1}^{n}\left[\lambda Q_{s}+V_{s}(\mathbf{Q})\right] \dot{q}_{s}(t) \varepsilon \Delta t+\mathrm{o}_{1}(\varepsilon) \geq \sum_{s=1}^{n}\left[\lambda \widetilde{Q}_{s}+V_{s}(\widetilde{\mathbf{Q}})\right] \dot{q}_{s}(t) \varepsilon \Delta t+\mathrm{o}_{2}(\varepsilon)
$$

where $\quad \mathrm{o}_{1}(\varepsilon), \mathrm{o}_{2}(\varepsilon)$ are infinitesimals of higher order of smallness, than $\varepsilon$. Up to an infinitesimal the minimum condition of the target functional is defined as follows:

$$
\sum_{s=1}^{n}\left[\lambda \widetilde{Q}_{s}+V_{s}(\widetilde{\mathbf{Q}})\right] \dot{q}_{s}(t) \geq \sum_{s=1}^{n}\left[\lambda Q_{s}+V_{s}(\mathbf{Q})\right] \dot{q}_{s}(t) .
$$

It follows from (17) that

$$
\max _{\widetilde{\mathbf{Q}} \in C^{[}\left[t_{0}, t_{1}\right]} \Phi=\max _{\tilde{\mathbf{Q}} \in C^{[}\left[t_{0}, t_{1}\right]} \sum_{s=1}^{n}\left[\lambda Q_{s}+V_{s}(\mathbf{Q})\right] \dot{q}_{s} .
$$

The conditions (14) and (18) allow to obtain the structure of solutions to the synthesis problem for an arbitrary number of degrees of freedom $n[4-8]$. 


\section{Synthesis of the intelligent fuzzy control based on the combined maximum principle}

For the dynamic system

$$
\dot{q}_{1}=q_{2}, \dot{q}_{2}=u, q_{1}(0)=20, q_{2}(0)=10
$$

it is required to synthesize the control $|u| \leq U$ that transfers the representative point of the phase space to the terminal point $(0,0)$ based on the condition of the minimum of the target functional

$$
J_{1}=\int_{0}^{t_{1}} d t \rightarrow \min .
$$

The optimal solution to the problem (19), (20) is obtained in [1].

It was shown in [2] that the deviation of the functional

$$
J_{1}=\int_{0}^{t_{1}} q_{1}^{2} d t \rightarrow \min
$$

on the trajectories of problem (19), (20) differs from the optimal value by not more than by $w$ if the transition from the optimal trajectory of A.T. Fuller occurs in the zone $\left|q_{1}\right| \leq \frac{1}{2}\left(\frac{w}{g}\right)^{\frac{2}{5}},\left|q_{2}\right| \leq \frac{1}{2}\left(\frac{w}{g}\right)^{\frac{1}{5}}$, where $g$ is the normalization constant, $w$ is a number. Thus in order to eliminate the mode with increasing switching, in this paper we find a quasioptimal solution to the performance problem in the space of bounded piecewise continuous functions for the dynamical system (19) based on the condition of minimum of the functional (21).

The use of the conditions of maximum for the generalized power function allows to obtain a quasi-optimal solution of the synthesis problem [5-8] on the boundary of the domain of admissible controls

$$
u_{1}\left(q_{1}, q_{2}\right)=\lambda^{-1}|U| \operatorname{sign}\left(-\frac{\lambda\left|q_{2}\right| q_{2}}{2\left|q_{1}\right|}-q_{1}\right)
$$

and within

$$
u_{2}\left(q_{1}, q_{2}\right)=\lambda^{-1}\left(-\frac{\lambda\left|q_{2}\right| q_{2}}{\left|q_{1}\right|+C}-2 q_{1}\right)
$$

where $C$ is constant.

We propose the method of control consisting of two stages. In the first stage, using the relay control law (22), the control object (19) is brought into the vicinity of the terminal state. The second stage uses continuous control (23).

The fuzzy instruction for solving the problem (19), (21) can be reduced to the rule: outside the neighborhood the relay control is used, inside it the continuous control is used. We propose the following structure of fuzzy control [7]

$$
u=\mu_{1}^{\prime}\left(q_{1}\right) u_{1}\left(q_{1}, q_{2}\right)+\mu_{2}^{\prime}\left(q_{1}\right) u_{2}\left(q_{1}, q_{2}\right)
$$

where $\mu_{1}^{\prime}, \mu_{2}^{\prime}$ are membership functions such as when moving away from the terminal point, $\mu_{1}^{\prime}$ decreases, but $\mu_{2}^{\prime}$ increases. 
For comparison, we choose the following membership functions [11]:

$$
\mu_{11}^{\prime}\left(q_{1}\right)=\frac{1}{1+e^{-\left(q_{1}-k\right)}}, \mu_{21}^{\prime}\left(q_{1}\right)=\frac{1}{1+e^{-\left(q_{1}-k\right)}},
$$

and $[7,10,11]$

$$
\mu_{12}^{\prime}=\frac{1}{q_{1}+1} A\left(q_{1}, k\right), \mu_{22}=\frac{q_{1}}{q_{1}+1} B\left(q_{1}, k\right), A\left(q_{1}, k\right)=\frac{k\left(q_{1}\right)\left(1+q_{1}\right)}{q\left(1+k\left(q_{1}\right)\right)}, B(q, k)=\frac{\left(1+q_{1}\right)}{1+k\left(q_{1}\right)},
$$

and the calculation of the parameter $k\left(q_{1}\right)$ is carried out according to the rule [7, 10]:

$$
k\left(q_{1}\right)=\left\{\begin{array}{cc}
k_{2}^{\prime}, & q_{1} \leq q_{1}^{\prime}, \\
\frac{q_{1}\left(k_{1}^{\prime}-k_{2}^{\prime}\right)+q_{2}^{\prime} k_{2}^{\prime}-q_{1}^{\prime} k_{1}^{\prime}}{q_{2}^{\prime}-q_{1}^{\prime},} & q_{1}^{\prime}<q_{1}<q_{2}^{\prime}, \\
k_{1}^{\prime}, & q_{1} \geq q_{2}^{\prime},
\end{array}\right.
$$

where $q_{1}^{\prime}, q_{2}^{\prime}$ are the parameters of the input linear Z-shaped and S-shaped membership functions, $k_{1}^{\prime}, k_{2}^{\prime}$ are the parameters of the output linear Z-shaped and S-shaped membership functions [11]. The input is the position relative to the terminal point, the output value is the value of the parameter $k\left(q_{1}\right)$.

The results of mathematical modeling are shown in Figures 2, 3, where the solid line denotes the curves corresponding to the control (24) with the membership functions (25), the dashed curve denotes curves corresponding to the optimal solution of L.S. Pontryagin, and the points denotes curves corresponding to the control (24) with membership functions (26). An analysis of the presented results lets us to conclude that the use of fuzzy quasioptimal control allows to avoid the mode with frequented switching. Intelligent fuzzy control (24) with membership functions (26) provides the time to reach the terminal point, which coincides within the selected viewing scale with the optimal time. The calculations show that the obtained result excludes the mode of frequented switching with a decrease in performance by $0.4 \%$ in comparison with the classical solution of L.S. Pontryagin, which confirms its proximity to the optimal solution.
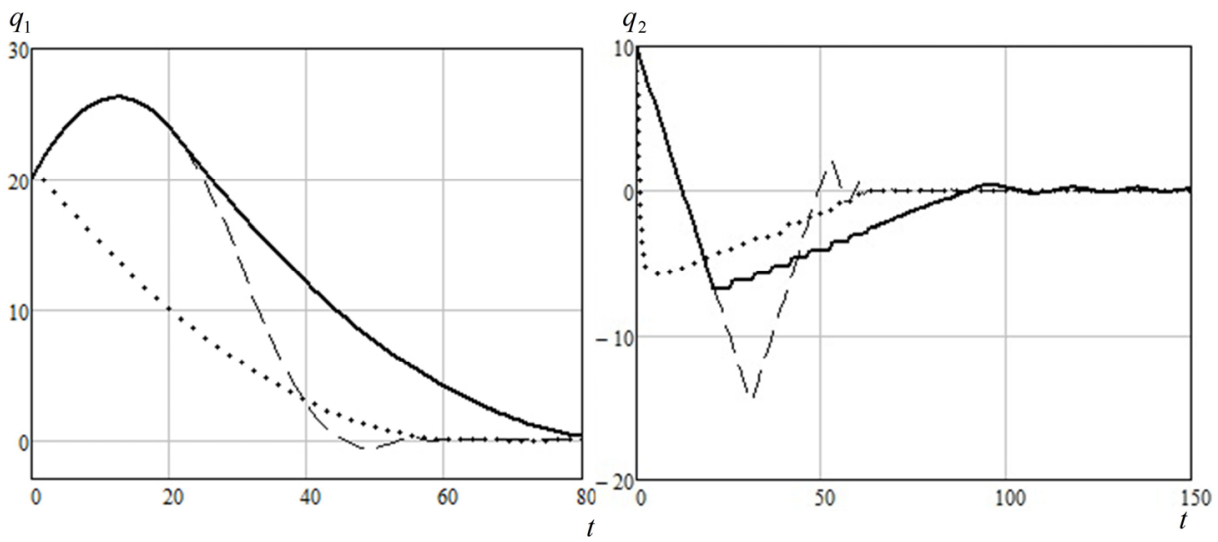

Fig. 2. The transient process. 


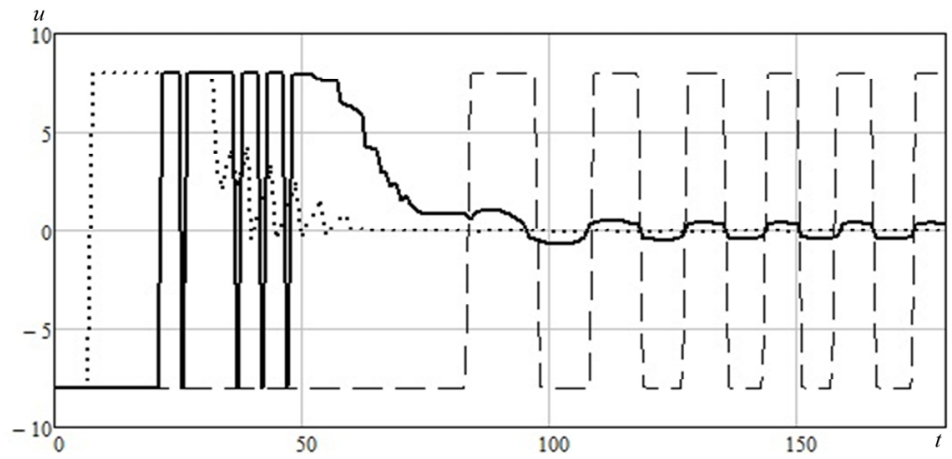

Fig. 3. The controls.

\section{Conclusion}

The solution of the problem of synthesis of optimal control using the HamiltonOstrogradskii principle led to the obtaining the new optimality conditions for the Lagrangian systems in the form of a maximum of the generalized power function on the set of bounded and continuous functions. Mathematical modeling confirmed the proximity of the results to the optimal results.

The paper has been accomplished with the support of Russian Federal Property Fund grants No. 1801-00385 A, № 18-08-01494 A.

\section{References}

1. L.S. Pontryagin, V.G. Boltyanskii, R.V. Gamkrelidze, E.F. Mishchenko The Mathematical Theory of Optimal Processes (USA: New York, 1986)

2. O.E. Maikova Suboptimal Regimes in the Fuller Problem. Proc. Steklov Inst. Math. 236, 214 (2002)

3. I.M. Anan'evskii, S.A. Reshmin, Journal of Computer and Systems Sciences International, 53, 473 (2014)

4. A.I. Lur'e, Analiticheskaya Mekhanika (Analytical mechanics) (Gos. Izd. Fiz.-Matem. Liter., Moscow, 1961)

5. A.A. Kostoglotov, S.V. Lazarenko, Journal of Communications Technology and Electronics, 62, 123 (2017)

6. A.A. Kostoglotov, A.I. Kostoglotov, S.V. Lazarenko, Automatic Control and Computer Sciences, 41, 274 (2007)

7. A. Kostoglotov, S. Lazarenko, I. Derabkin, A. Kuzin, I. Pugachev, O. Manaenkova, Advances in Intelligent Systems and Computing, 679, 321 (2017)

8. A.A. Kostoglotov, S.V. Lazarenko, D.S. Andrashitov, I.V. Deryabkin MATEC Web Conf, 77, 15002 (2016)

9. E.S. Pyatnitskiy, Automation and Remote Control, 50, 64 (1989)

10. N.YU. Muzychenko, Journal of Communications Technology and Electronics, 55, 755 (2010)

11. S.A. Orlovskij The problem of decision making with fuzzy initial information (Nauka, Moscow 1981) 